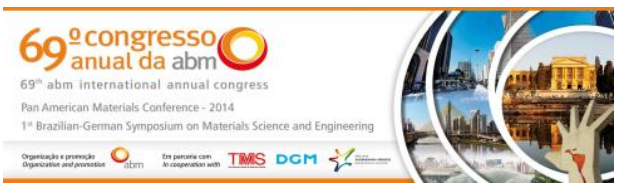

Tema: Corrosão

\title{
SUSCEPTIBILIDADE À CORROSÃO DE DOIS DIFERENTES TIPOS DE AÇO (CA-50 E CA-60) UTILIZADOS NA CONSTRUÇÃO CIVIL*
}

\author{
Luís Gustavo Costa de Castro ${ }^{1}$ \\ Marinalda Claudete Pereira ${ }^{2}$ \\ Eduardo Norberto Codaro ${ }^{3}$ \\ Heloisa Andréa Acciarit
}

\section{Resumo}

Neste trabalho foram utilizadas amostras dos aços CA-50 e CA-60 utilizados na construção civil, para avaliar a corrosão pelos íons cloreto. Para tanto, medidas eletroquímicas foram realizadas em soluções de $\mathrm{NaCl} 2,0 \mathrm{~g} / \mathrm{L}$ e $4,0 \mathrm{~g} / \mathrm{L}$, naturalmente aeradas. Verificou-se um processo de corrosão generalizada dos aços CA-50 e CA-60 nas duas concentrações avaliadas e uma forte influência do aumento da concentração no comportamento corrosivo de ambos os tipos de aço. Neste estudo comparativo, verificou-se que, além dos perfis potenciodinâmicos serem similares, a resistência de polarização conduziu a resultados da mesma ordem de magnitude, concluindo-se que não existe uma vantagem significativa ao uso de um em relação ao outro, no que se refere à resistência à corrosão.

Palavras-chave: Aços CA-50 e CA-60; Cloreto; Técnicas Eletroquímicas; Corrosão.

\section{SUSCEPTIBILITY TO CORROSION OF TWO DIFFERENT TYPES OF STEEL (CA- 50 AND CA-60) USED IN CIVIL CONSTRUCTION}

\section{Abstract}

The aim of the present study was to investigate de corrosion resistance in chloride medium of two cylindrical steel samples from civil construction (CA-50 and CA-60). For this purpose, electrochemical measurements were performed in $\mathrm{NaCl}$ solution, 2.0 and $4.0 \mathrm{~g} / \mathrm{L}$, naturally aerated. According to electrochemical responses, it was observed a generalized corrosion process in the two concentrations and a strong influence of increasing concentration on the corrosion behavior of both types of steel used. In this comparative study, it was established that, besides the potentiodynamic profiles are quite similar, the estimated polarization resistance resulted in slightly different results, but within of the same order of magnitude. It was concluded, therefore, that there is no significant advantage in using one instead of the other one regarding to corrosion resistance.

Keywords: Steels CA-50 and CA-60; Chloride; Electrochemical Techniques; Corrosion.

1 Aluno de Engenharia Civil, Faculdade de Engenharia de Guaratinguetá, Unesp, Guaratinguetá, $S P$, Brasil.

2 Pós-Doutorado, Engenharia Química, Pesquisador, Faculdade de Engenharia de Guaratinguetá, Unesp, Guaratinguetá, SP, Brasil.

3 Doutor em Química, Professor Adjunto, Faculdade de Engenharia de Guaratinguetá, Unesp, Guaratinguetá, SP, Brasil.

4 Doutora em Química, Professora Assistente Doutora, Faculdade de Engenharia de Guaratinguetá, Unesp, Guaratinguetá, SP, Brasil.

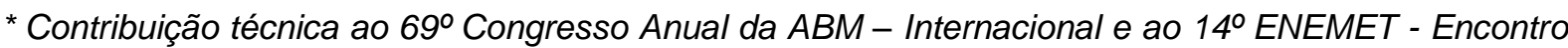
Nacional de Estudantes de Engenharia Metalúrgica, de Materiais e de Minas, 21 a 25 de julho de 2014, São Paulo, SP, Brasil.
} 


\section{INTRODUÇÃO}

Os processos corrosivos têm um grande impacto no desempenho e na durabilidade das estruturas de concreto. As perdas financeiras ocasionadas pelos processos de degradação e corrosão das estruturas metálicas e de concreto são, em geral, muito elevadas [1]. Nos Estados Unidos o custo anual da corrosão chega a 3,1\% do PIB, o que totaliza US $\$ 276$ bilhões, contra 3,5\% no Brasil, sem contar os custos indiretos, como interrupções no funcionamento de estabelecimentos industriais, contaminação de produtos, problemas de segurança (incêndios, explosões, liberação de produtos tóxicos, etc.), acidentes pessoais, entre outros. Isto tem motivado amplo desenvolvimento da pesquisa neste campo da Engenharia [2].

Os altos custos que envolvem o reparo ou a perda de um material são alguns dos aspectos que tornam a corrosão de armaduras um grande problema para 0 profissional de engenharia civil. Uma questão pertinente a este respeito refere-se ao fato de que as construções do passado eram mais robustas, isto é, as largas espessuras de cobrimento forneciam à estrutura um caráter mais protetor e, consequentemente maior durabilidade. Com o decorrer do tempo, a evolução dos materiais e das metodologias de cálculo concebeu um caráter mais esbelto a um menor custo, entretanto, proporcionou menor durabilidade às construções [3-5].

Os aços das armaduras geralmente estão protegidos da corrosão pela formação de um complexo filme pseudopassivo de óxidos de ferro promovido pela própria alcalinidade do concreto [6,7]. Entretanto, agentes promotores ou causadores da corrosão, como o dióxido de carbono e os íons cloreto, incorporados durante a etapa de preparação do concreto, ou provenientes do meio ambiente, podem romper a camada pseudopassiva conduzindo à redução da secção transversal de reforço (vergalhão) pela formação de trincas na cobertura externa de concreto [6-13].

O dióxido de carbono pode difundir-se no concreto através dos poros e reagir com as substâncias alcalinas originando uma redução do $\mathrm{pH}$ local. A reação de carbonatação inicia-se na parte exterior da armadura e pode atingir a camada pseudopassiva, comprometendo sua estabilidade e conduzindo à corrosão generalizada do aço, formando óxidos hidratados e hidróxidos de ferro como produtos de corrosão, que apresentam um volume significativamente maior que 0 próprio átomo de ferro que lhes deu origem, exercendo deste modo, uma pressão sobre a cobertura de concreto, que pode provocar fissuras e até desprendimento da armadura. A velocidade com que esta reação ocorre depende da espessura e qualidade do recobrimento, do tipo de cimento e do teor de umidade do concreto [4-6]. A introdução de íons cloreto no concreto, por outro lado, pode ocorrer durante a sua fabricação quando aparece como contaminante do cimento, ou devido à natureza da água utilizada na mistura [4-6].

Estas considerações visam ressaltar a importância dos estudos desses fatores no problema da proteção das armaduras de concreto, como aspectos inerentes à engenharia preventiva e corretiva. Neste sentido, o destaque é dado às técnicas eletroquímicas, uma vez que a corrosão é um fenômeno eletroquímico. Quando esta análise é combinada com o conhecimento das propriedades básicas dos concretos, possibilita maiores detalhes nas inspeções das estruturas e nas pesquisas em nível laboratorial.

Uma vez depassivada a armadura, para as mesmas condições dadas pelo tipo de concreto e ambiente, as velocidades de corrosão serão diferenciadas em função do tipo de aço empregado. Dependendo do processamento durante a fabricação e do teor de carbono, um determinado tipo de aço pode ser mais suscetível à corrosão

* Contribuição técnica ao 69 Congresso Anual da ABM - Internacional e ao 14ํㅡㄹ ENEMET - Encontro Nacional de Estudantes de Engenharia Metalúrgica, de Materiais e de Minas, 21 a 25 de julho de 2014, São Paulo, SP, Brasil. 


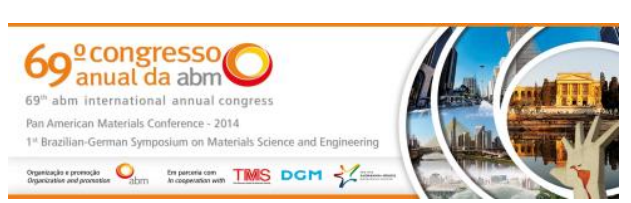

em comparação com outros, de menor dureza e de mais baixa resistência mecânica. CA-50 e CA-60 são as categorias destes aços-carbono. O termo CA é uma abreviatura de concreto armado. No ensaio de tração, os valores mínimos de resistência de escoamento, ou seja, a carga de trabalho que o vergalhão deve suportar define esta classificação: $50 \mathrm{kgf} / \mathrm{mm}^{2}$ ou $500 \mathrm{MPa}$ para o CA-50 e 60 $\mathrm{kgf} / \mathrm{mm}^{2}$ ou $600 \mathrm{MPa}$ para o CA-60.

Sendo assim, neste trabalho propõe-se avaliar o efeito da concentração de íons cloreto na resistência à corrosão de dois tipos de aço utilizados com o mesmo propósito na construção civil, CA-50 e CA-60, utilizando-se técnicas eletroquímicas e de análises de superfície.

Este estudo representa parte de um projeto em desenvolvimento, que consiste em investigar os efeitos da espessura de discos de argamassa, preparados a partir de diferentes razões água/cimento $(0,45$ e 0,65$)$, na resistência à corrosão do aço em meio de cloreto. Nesta primeira etapa, ênfase foi dada ao comportamento eletroquímico para definir o tipo de aço mais resistente à corrosão pelos íons cloreto. Para completar este estudo, posteriormente serão preparados discos de argamassa contendo o aço escolhido (aquele com o melhor desempenho na primeira etapa) moldado em concreto, utilizando-se Cimento Portland Ordinário (OCP), o qual será posicionado na base de uma célula eletroquímica para repetição dos ensaios eletroquímicos de corrosão, considerando estas novas variáveis.

\section{MATERIAIS E MÉTODOS}

Amostras cilíndricas de vergalhões dos aços, CA-50 (1,6 cm de diâmetro) e CA60 (0,5 cm de diâmetro) foram polidas com lixas de SiC (80-1200) previamente ao seu uso nos ensaios eletroquímicos. Para avaliar a corrosão pelos íons cloreto, medidas eletroquímicas foram realizadas em soluções de $\mathrm{NaCl}$, de 2,0 e 4,0 g/L naturalmente aeradas. Utilizou-se uma célula eletroquímica convencional, própria para eletrodos de trabalho planos. A superfície polida do aço constituiu o eletrodo de trabalho; como eletrodo auxiliar foi utilizada uma rede de $\mathrm{Pt}$ e; como referência, o eletrodo $\mathrm{Ag} / \mathrm{AgCl}$. Medidas de potencial em circuito aberto com o tempo (OCP) e de polarização potenciodinâmica (Tafel e polarização cíclica-CP, além da espectroscopia de impedância eletroquímica-EIS), foram realizadas por meio de um potenciostato/galvanostato Autolab (Eco. Chemie B.V., Utrecht, Netherlands), modelo PGSTAT302.

\section{RESULTADOS E DISCUSSÃO}

Na Figura 1 estão apresentadas as curvas de OCP obtidas para os aços CA-50 e CA-60 em duas concentrações de $\mathrm{NaCl}, 2,0$ e 4,0 g/L. A avaliação do potencial em circuito aberto possibilita estabelecer uma comparação da corrosividade do material em diferentes meios, de modo que, quanto maior for o valor deste potencial, maior será a sua resistência à corrosão no meio considerado. Neste sentido, CA-60 apresentou potenciais mais positivos, em comparação ao CA-50, e também foi o que mostrou menor influência ao aumento da concentração dos íons $\mathrm{Cl}^{-}$, uma vez que, para CA-50 esta variação na concentração promoveu uma diferença de aproximadamente $100 \mathrm{mV}$ nos valores de OCP próximos do estado estacionário, indicando que a mudança na concentração tornou o meio ainda mais oxidante para CA-50. Em ambas as condições, um comportamento típico de estado ativo foi

\footnotetext{
* Contribuição técnica ao $69^{\circ}$ Congresso Anual da ABM - Internacional e ao 14ํㅡㄹ ENEMET - Encontro Nacional de Estudantes de Engenharia Metalúrgica, de Materiais e de Minas, 21 a 25 de julho de 2014, São Paulo, SP, Brasil.
} 


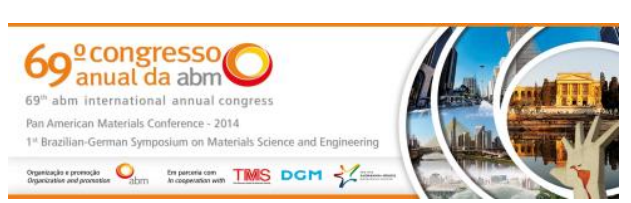

observado, pelo potencial descendente com o tempo, durante o período de monitoramento do potencial em circuito aberto.

Nenhuma diferença substancial foi observada comparando-se os perfis potenciodinâmicos obtidos para CA-50 e CA-60, tanto no que diz respeito à região de Tafel, como a espectros mais amplos de potencial que compreendem a varredura anódica, Figura 2.

Nas respectivas regiões catódicas destas curvas, a corrente catódica não foi totalmente estacionária. Esta variação em geral é devida às mudanças na composição química da superfície durante a polarização e conduz, como consequência, a um comportamento eletroativo mais acentuado nos ramos anódicos.
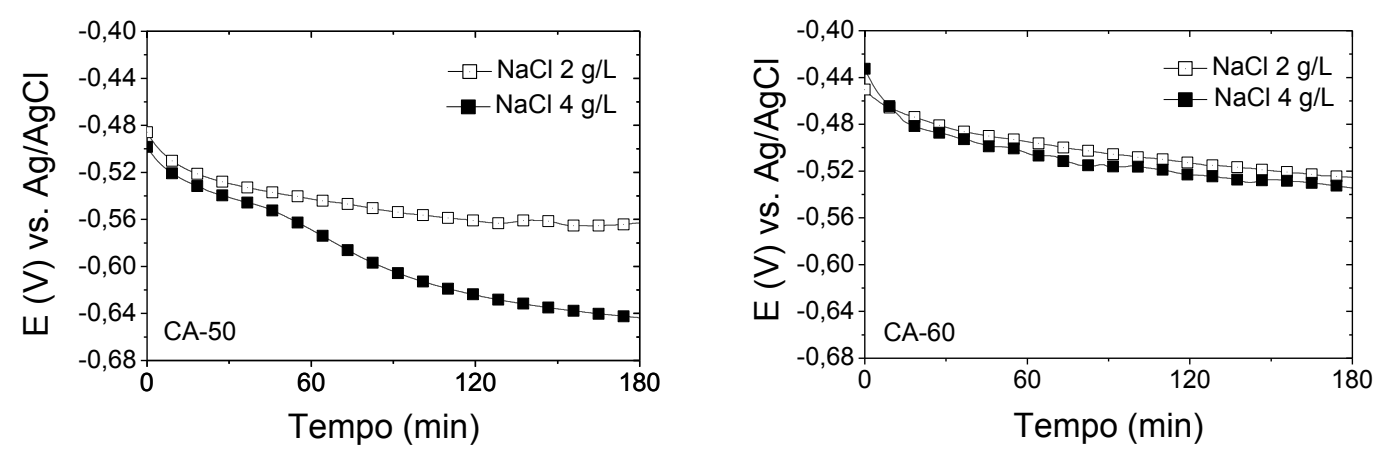

Figura 1. Curvas de OCP dos aços CA-50 e CA-60 em meios de $\mathrm{NaCl}(2,0$ e 4,0 g/L).
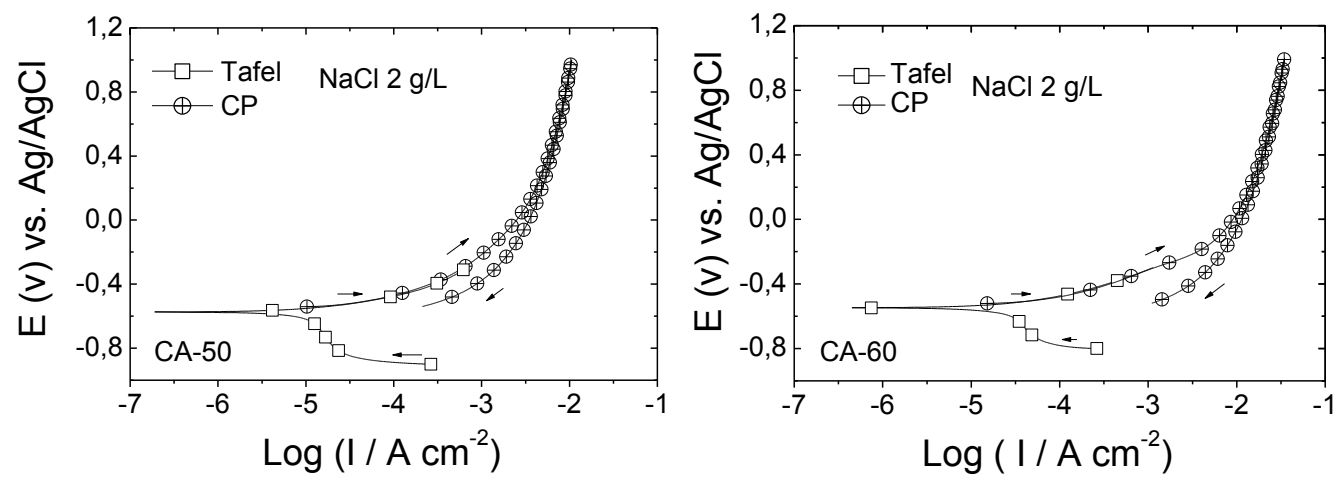

Figura 2. Curvas de Tafel e de CP dos aços CA-50 e CA-60 em meio de $\mathrm{NaCl}$ 2,0 g/L.

Para o aço, o mecanismo eletroquímico do processo corrosivo origina-se em inúmeras células de ação local da superfície metálica [9]. $\mathrm{Na}$ área anódica, inicia-se com a oxidação do ferro segundo a Equação 1:

$\mathrm{Fe}(\mathrm{s}) \rightarrow \mathrm{Fe}^{2+}(\mathrm{aq})+2 \mathrm{e}^{-}$

Considerando o meio neutro e aerado, a reação catódica pode ser representada pela seguinte Equação 2:

$\mathrm{H}_{2} \mathrm{O}(\mathrm{l})+1 / 2 \mathrm{O}_{2}(\mathrm{~g})+2 \mathrm{e}^{-} \rightarrow 2 \mathrm{OH}^{-}(\mathrm{aq})$

Como produto de corrosão tem-se o hidróxido de ferro (II), $\mathrm{Fe}(\mathrm{OH})_{2}$, que em meio aerado transforma-se lentamente em hidróxido de ferro (III), $\mathrm{Fe}(\mathrm{OH})_{3}$, castanhoalaranjado, que é também escrito sob a forma de $\mathrm{Fe}_{2} \mathrm{O}_{3} \cdot \mathrm{nH}_{2} \mathrm{O}$. Portanto, a coloração alaranjada tão desagradável que aparece no concreto indica que a armadura está sofrendo corrosão com uma intensidade que pode ser relacionada com a extensão dessa coloração [9].

\footnotetext{
* Contribuição técnica ao $69^{\circ}$ Congresso Anual da ABM - Internacional e ao 14ํㅡㄹ ENEMET - Encontro Nacional de Estudantes de Engenharia Metalúrgica, de Materiais e de Minas, 21 a 25 de julho de 2014, São Paulo, SP, Brasil.
} 


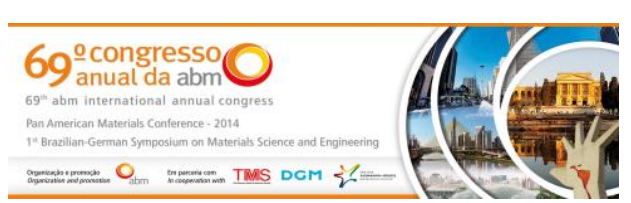

Os aços-carbono comuns contêm mais de $97 \%$ de $\mathrm{Fe}$, até $2 \%$ de $\mathrm{C}$ e outros elementos remanescentes do processo de fabricação. $\mathrm{O}_{2} \mathrm{O}_{2}(\mathrm{~g})$ e a $\mathrm{H}_{2} \mathrm{O}(\mathrm{l})$ constituem o meio no qual os materiais estão mais frequentemente expostos. Como os potenciais-padrão de redução do $\mathrm{Fe}^{2+}(\mathrm{aq}) / \mathrm{Fe}(\mathrm{s})\left[\mathrm{E}^{0}=-0,44 \mathrm{~V}\right]$ e do $\mathrm{Fe}^{3+}(\mathrm{aq}) / \mathrm{Fe}^{2+}(\mathrm{aq})$ $\left[\mathrm{E}^{0}=0,77 \mathrm{~V}\right]$ são menores que aquele para a redução de $\mathrm{O}_{2}(\mathrm{~g})\left[\mathrm{E}^{0}=1,23 \mathrm{~V}\right]$, o $\mathrm{Fe}(\mathrm{s})$ pode ser oxidado pelo $\mathrm{O}_{2}(\mathrm{~g})$. Para que essas reações ocorram de forma simultânea, a transferência de elétrons tem que ser através do aço, desde uma região onde acontece a oxidação do $\mathrm{Fe}(\mathrm{s})$ (anódica) até outra onde acontece a redução do $\mathrm{O}_{2}(\mathrm{~g})$ (catódica), como nas pilhas. O produto de corrosão resultante é a ferrugem, cuja formação pode ser sucintamente representada pela equação 3 .

$4 \mathrm{Fe}(\mathrm{s})+3 \mathrm{O}_{2}(\mathrm{~g})+2 \mathrm{nH}_{2} \mathrm{O}(\mathrm{l}) \rightarrow 2 \mathrm{Fe}_{2} \mathrm{O}_{3} \cdot \mathrm{nH}_{2} \mathrm{O}(\mathrm{s}) \quad \mathrm{n}=1,2,3$

A histerese positiva nas curvas de polarização anódica (Figura 2) indica ausência de passivação na varredura reversa, denotando um processo de corrosão generalizada. $\mathrm{Na}$ faixa de pH investigada (entre 5,0 e 6,0 antes e após os ensaios de corrosão) as reações anódicas envolvem a formação de íons complexos de $\mathrm{Fe}$ (II) e $\mathrm{Fe}$ (III) e possível precipitação de hidróxido de ferro (III) [9].

Os gráficos de EIS medidos nos potenciais de circuito aberto e apresentados no formato do plano complexo, Figura 3, exibiram significativa diminuição no arco capacitivo com o aumento da concentração dos íons cloreto para os dois tipos de aço avaliados. Entretanto diferenças observadas no aspecto dos semicírculos obtidos mostram que CA-50 e CA-60 apresentam comportamentos ligeiramente distintos no potencial de circuito aberto. O diâmetro do arco capacitivo foi consideravelmente menor para CA-60, o que denota uma estimativa de $R_{\mathrm{p}}$, a resistência de polarização, menor neste último caso. Apesar disso, os valores obtidos (algumas unidades de $\mathrm{k} \Omega \mathrm{cm}^{2}$ ) correspondem à mesma ordem de magnitude.
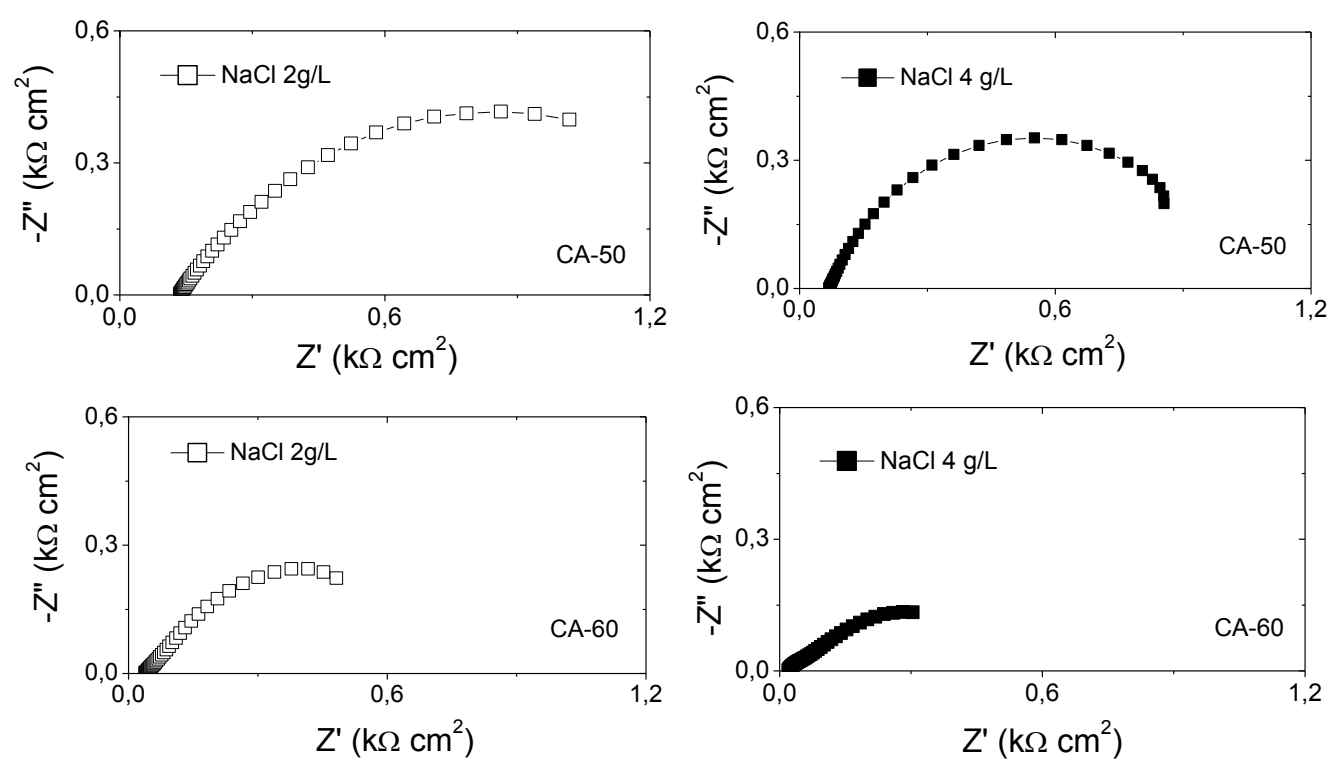

Figura 3. Espectros de EIS obtidos no plano complexo para os aços CA-50 e CA-60 em meios de $\mathrm{NaCl}(2,0$ e $4,0 \mathrm{~g} / \mathrm{L})$.

\section{CONCLUSÃO}

Verificou-se pela resposta eletroquímica um processo de corrosão generalizada dos aços CA-50 e CA-60 nas duas concentrações de íons cloreto avaliadas. Além dos

* Contribuição técnica ao 69 Congresso Anual da ABM - Internacional e ao 14ํㅡㄹ ENEMET - Encontro Nacional de Estudantes de Engenharia Metalúrgica, de Materiais e de Minas, 21 a 25 de julho de 2014, São Paulo, SP, Brasil. 
